\title{
Prevalence of G6PD deficiency in Iranian neonates with jaundice: a systematic review and meta-
}

Javadi M. ${ }^{\text {a, }}$, analysis

Deravi $S_{.}^{b}$,

Zarei S. ${ }^{\mathrm{b}}$,

Mahdavi N. ${ }^{\mathrm{c}}$,

Ranjbaran M., d,e

a Children Growth Research Center, Research Institute for Prevention of Non-Communicable Diseases, Qazvin University of Medical Sciences, Qazvin, Iran

b Department of Nutrition, School of Health, Qazvin University of Medical Sciences, Qazvin, Iran

c Department of Epidemiology, School of Public Health, Iran University of Medical Sciences, Tehran, Iran

d Department of Epidemiology, School of Public Health, Shahid Beheshti University of Medical Sciences,

Tehran, Iran

e Department of Epidemiology and Biostatistics, School of Public Health, Tehran University of Medical Sciences,

Tehran, Iran

\section{Abstract}

Objectives: This systematic review and meta-analysis study aimed to estimate the overall prevalence of Glucose-6-phosphate dehydrogenase (G6PD) deficiency in neonates with jaundice who were admitted to hospitals in Iran. Materials and methods: In this systematic review and metaanalysis, we searched PubMed/Medline, Scopus, ISI Web of Sciences, and Iranian Local databases up to December 2019.We calculated Prevalence and 95\% Confidence Interval (95\% CI) of G6PD deficiency as summary measures. We conducted subgroup analysis based on the sex and quality of studies, while meta-regression were applied for investigating the effect of years of studies and years of publication on the pooled prevalence. We applied sensitivity analysis to investigate the effect of excluding each study on the pooled prevalence estimation. Results: The total sample size was 9799 people. The pooled prevalence of G6PD deficiency among neonates with jaundice in Iran was 7.0\% (95\% CI: 5.5-8.5\%). The results of subgroup analysis showed that, pooled prevalence of G6PD deficiency among male neonate $(12.1 \%$, 95\%CI: 7.6-16.7\%) was more prevalent that female (3.00\%, 95\% CI: 1.1-4.9\%). Based on the sensitivity analysis, lower and higher pooled prevalence of G6PD deficiency was observed 5.8\% (95\%CI: 4.7-6.9\%) and 7.3\% (95\%CI: 5.7-8.8\%) respectively by excluding each study. Conclusion: The overall prevalence of G6PD deficiency was $7 \%$ in Iranian neonates with Jaundice. Prevalence was high in male and early hours of life. We recommend screening test for G6PD deficiency in neonates to prevent its complications. (C) 2021 Informa UK Limited, trading as Taylor \& Francis Group. 


\section{Author keywords}

deficiency; Glucosephosphate dehydrogenase; jaundice; meta-analysis; neonatal 